20

\title{
Ранняя диагностика меланомы кожи с применением нескольких изображающих систем
}

\author{
() К.Г. Кудрин ${ }^{1}$, Е.Н. Римская ${ }^{2}$, И.А. Аполлонова ${ }^{2}$, А.П. Николаев ${ }^{2}$, Н.В. Черномырдин ${ }^{2,3}$, \\ Д.С. Святославов ${ }^{4}$, Д.В. Давыдов ${ }^{1}$, И.В. Решетов ${ }^{1,4,9}$ \\ ${ }^{1}$ Академия постдипломного образования ФГБУ ФНКЦ ФМБА России, \\ 125371 Москва, Россия \\ ${ }^{2}$ Московский государственный технический университет им. Н.Э. Баумана, \\ 105005 Москва, Россия \\ ${ }^{3}$ Институт общей физики им. А.М. Прохорова РАН, \\ 119991 Москва, Россия \\ ${ }^{4}$ Институт регенеративной медицины, Первый Московский государственный медицинский университет им. \\ И.М. Сеченова, \\ 119991 Москва, Россия \\ e-mail: kudrin_k@rambler.ru, reshetoviv@mail.ru
}

Поступила в редакцию 10.12.2019 г.

В окончательной редакции 03.02.2020 г.

Принята к публикации 28.02.2020 г.

Предложен комплексный подход к ранней диагностике меланомы кожи. Подход предусматривает поэтапное обследование пигментных новообразований с помощью нескольких изображающих систем. Рассмотрены особенности морфометрии клинических изображений пигментных новообразований кожи, особенности изображающих систем, основные этапы автоматизированной обработки изображений и распознавания образов при диагностике меланомы. Показаны метрологические особенности предлагаемого подхода: ошибки определения клинических параметров новообразований кожи предлагаемыми методами не превышают допустимые ошибки. В результате апробации предложенного подхода установлено, что чувствительность и специфичность используемых методов превышает $90 \%$.

Ключевые слова: изображающие системы, пигментные новообразования кожи, ранняя диагностика меланомы, цифровая обработка изображений, машинное обучение.

DOI: $10.21883 /$ OS.2020.06.49416.53-20

\section{Введение}

Одним из самых опасных заболеваний кожи является меланома. Доля меланомы в структуре опухолевых заболеваний кожи невелика и в среднем по различным регионам мира составляет $3-5 \%[1,2]$. Однако меланома остается главной причиной смерти онкологических больных [3]. За последние два десятилетия заболеваемость меланомой возросла $[1,4,5]$. Стандартизированные показатели заболеваемости меланомой кожи ежегодно увеличиваются на 3-7\% [6-9]. Остро стоит проблема ранней диагностики меланомы, что подтверждается показателями пятилетней выживаемости больных [10]. Ошибки диагностики и соответственно ошибочное лечение могут приводить к развитию злокачественной опухоли $[9,11]$.

Наиболее точным методом диагностики злокачественных новообразований является морфологическое исследование удалённых биотканей (эксцизионная биопсия опухоли) [2-7]. На ранних стадиях патологии клинические признаки недостаточно выражены и необходима дифференциальная уточняющая диагностика, с которой без инструментальных средств способны справиться только высококвалифицированные медицинские работники [5,8]. Это приводит к ошибкам диагностики и избыточному травмированию пациентов. Наиболее информативными признаками онкопатологии являются изменения размеров, формы и цвета новообразований кожи, которые удается обнаружить только при регулярном обследовании, что возможно только при проведении регулярного скрининга. Исключить субъективный фактор и повысить точность диагностики при скрининге могут автоматизированные системы для скрининга пигментных новообразований кожи [12]. Обнаруживаемые при скрининге подозрительные новообразования должны подвергаться дополнительному обследованию с помощью различных инструментальных методов.

В онкодерматологии наиболее распространён визуальный осмотр [4,13]. Для неинвазивной уточняющей диагностики новообразований кожи наиболее часто применяются следующие методы и соответствующие им инструментальные средства: цифровая дерматоскопия [1321], магнитно-резонансная микроскопия [22], конфокальная лазерная микроскопия [23-26], оптическая когерентная томография [27-30], ультразвуковое сканирование кожи [31-33], термометрия [34-36], флуоресцентная диагностика $[37,38]$, спектроскопия и визуализация в терагерцовом диапазоне $[39,40])$. 
Перечисленные методы и средства неинвазивной диагностики новообразований кожи обладают своими достоинствами и недостатками. Дерматоскопия имеет высокую чувствительность и специфичность (92.4 и 93.7\% соответственно) [13-21], однако этот метод требует специальных знаний. Флуоресцентная диагностика имеет недостаточную для дифференциальной диагностики чувствительность и специфичность $(87.2 \%, 94.8 \%)$ [37,38]. Высокочастотное ультразвуковое сканирование кожи не позволяет проводить дифференциальную диагностику из-за схожести эхографических признаков доброкачественных и злокачественных новообразований [31-33]. Исследования по применению методов конфокальной лазерной микроскопии, оптической когерентной томографии, спектроскопии и визуализации в терагерцовом диапазоне стали появляться сравнительно недавно и имеющиеся результаты пока не позволяют говорить об их диагностической эффективности по сравнению с другими методами [23-30,39,40]. Термография имеет богатую историю применения в диагностике онкологических заболеваний. Гистологически диагноз подтверждается в $94.8 \%$ случаев при меланоме кожи и только в $67.9 \%$ при доброкачественных новообразованиях кожи [34-36].

Значительный прогресс в развитии инструментальных средств способствует повышению эффективности ранней диагностики меланомы. Этому также способствует применение современных технологий обработки изображений и распознавания образов. Современная элементная база для оптической визуализации существенно подешевела и повысила свою эффективность. Выросли возможности обработки изображений в реальном масштабе времени [41-43]. Также системы визуализации доступны на большинстве мобильных платформ (смартфоны и планшеты).

В настоящей работе предлагается перспективный подход к раннему выявлению меланомы кожи на основе комбинирования нескольких диагностических каналов в рамках некоторой последовательности их применения. При этом используются методы вычислительной диагностики, позволяющие анализировать разнородную диагностическую информацию и повысить эффективность постановки диагноза.

В клинической практике накоплен значительный опыт диагностики новообразований кожи с использованием визуальных клинических признаков. Современная элементная база позволяет создать изображающие системы в видимом диапазоне. Учитывая, что одним из эффективных методов раннего обнаружения меланомы кожи является скрининг пигментных новообразований [44], целесообразно использовать современные мобильные устройства с цифровыми камерами для самостоятельного предварительного обследования населения. Прообразы таких систем появляются, и в настоящее время уже доступны мобильные приложения, помогающие пользователю в проведении самообследования [45]. Для построения полноценной мобильной системы скрининга таким приложениям не хватает количественной оценки визуальных признаков новообразований кожи, а также метрологического обеспечения измерений. Особенности такой системы мы рассмотрим в настоящей работе.

После самообследования в случае обнаружения подозрительного новообразования кожи пациенту необходимо обратиться в профильное медицинское учреждение. В популяции всё чаще встречаются люди с синдромом кожной полиневоплазии (множественные пигментные образования кожи, 20 и более). Таких пациентов сложно обследовать с помощью большинства существующих инструментальных средств. Необходимо применение систем скрининга пигментных новообразований пациента [12]. Такие системы уже используются в диагностической практике [46]. В основе их работы лежит регистрация изображений поверхности кожи в видимом диапазоне и методы распознавания образов. Однако отсутствие метрологического обеспечения ограничивает возможности таких систем. В работе будут рассмотрены особенности системы скрининга пигментных новообразований пациента.

После скрининга в лечебном учреждении обнаруженные подозрительные новообразования кожи, которые не удалось однозначно диагностировать, необходимо обследовать дополнительно. Это может потребоваться, например, для дифференциальной диагностики меланомы на ранней стадии и её предшественников (диспластический невус, злокачественное лентиго). Большинство описанных выше инструментальных средств предназначены для такого дополнительного обследования. Чаще всего используют дерматоскопию. Высокую чувствительность и специфичность дерматоскопии при диагностике меланомы (92.4\% и 93.7\% соответственно) [13-21] обеспечивает только значительный опыт диагноста, тогда как менее опытному специалисту сложно отличить меланому от её предшественников. Мы предлагаем дополнительное автоматизированное термографическое обследование, не требующее специальных навыков, что также будет рассмотрено в настоящей работе.

\section{1. Морфометрия клинических изображений пигментных новообразований кожи}

Клинические описания новообразований кожи дают сведения о размерах, форме, цвете новообразований кожи, особенностях границы, текстуры поверхности [47]. Методики, используемые в клинической практике для раннего выявления меланомы (например, правило $\mathrm{ABCD}$ ), позволяют экспертно (в баллах) оценить размеры (диаметр), форму (асимметрию), цвет (неоднородность), особенности границы (резкость, изрезанность). Кроме того, в клинической практике известен перечень визуальных признаков малигнизации пигментных новообразований кожи [48]:

- исчезновение кожного рисунка на поверхности;

- появление блестящей, глянцевой поверхности; 

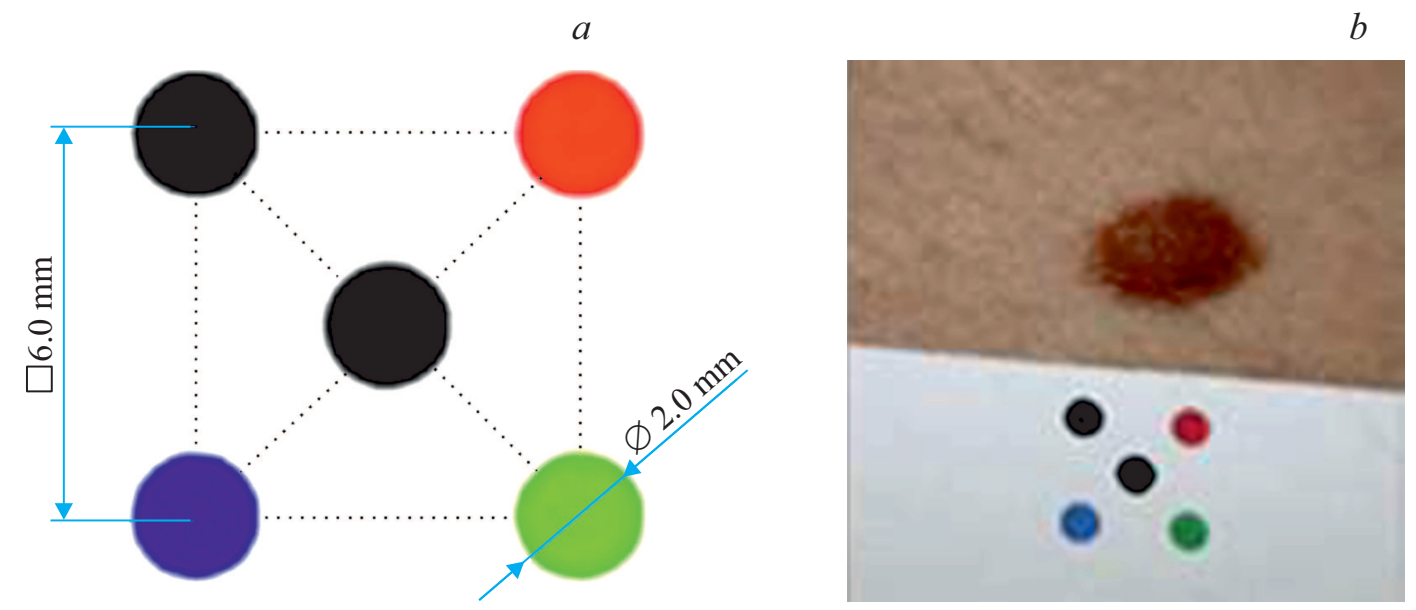

Рис. 1. Калибровочный эталон. $a-$ модель эталона, $b$ - зарегистрированное изображение новообразования с эталоном.

- появление асимметрии или неправильности очертаний, изменение формы;

- появление изрезанности границы;

- горизонтальный рост;

- шелушение поверхности с образованием сухих корочек;

- отсутствие или выпадение волосяного покрова на поверхности;

- частичное (неравномерное) или полное изменение окраски, появление участков депигментации;

- появление мелких узелков на поверхности;

- вертикальный рост над окружающими тканями.

Визуальные признаки и признаки озлокачествления группируются по категориям, описывающим размеры, форму, границу, цвет и равномерность пигментации, изменения размеров, формы, границы, цвета и равномерности пигментации. В табл. 1 приведён комплекс клинических параметров, с помощью которого возможно описание пигментных новообразований кожи и который можно определять автоматизированно с помощью методов морфометрии медико-биологических изображений.

За исключением размеров новообразований кожи (диаметр, площадь), все клинические параметры являются качественными. Для их автоматического определения необходим комплекс измеряемых параметров. Взаимосвязь комплекса клинических и измеряемых параметров показана в табл. 2 .

На основе доступных в литературе данных о размерах новообразований кожи и их диагностически значимых изменениях с помощь методологии расчёта погрешностей косвенных измерений были получены значения допустимых погрешностей измерения линейных параметров и параметров площади. Для оценки допустимых погрешностей измерения параметров цвета использовались современные данные о возможностях зрительного анализатора человека (табл. 3).

Таким образом, мы определили комплекс измеряемых параметров, требования к допустимым погрешностям их измерения при морфометрии изображений пигментных новообразований кожи.

\section{2. Изображающие системы для скрининга и диагностики новообразований кожи}

\section{1. Мобильная система скрининга пигментных новообразований кожи}

Обычно человек не может проводить самостоятельный скрининг своих пигментных новообразований кожи, так как не обладает необходимыми знаниями. Для самостоятельного скрининга можно использовать специальное программное обеспечение, установленное на мобильном устройстве с цифровой камерой (смартфон, планшет). Специальное программное обеспечение регистрирует клинические изображения, сегментирует их, обнаруживает пигментное новообразование, измеряет клинические параметры и даёт заключение о необходимости обратиться к профильному специалисту.

Отличительной особенностью разработанной нами системы $[49,50]$ является использование калибровочного эталона, который распечатывает пользователь перед обследованием новообразования (рис. 1). Эталон необходимо размещать вблизи от новообразования, чтобы он попадал в кадр. Эталон содержит палитру цветовой системы RGB (R - red; G - green; B - blue) и шкалу размеров. Изображение эталона регистрируется камерой одновременно с новообразованием, что позволяет скорректировать передачу цветовых характеристик новообразования в различных условиях съемки и определить его истинные размеры. Программное обеспечение оценивает качество изображения, выполняет распознавание и обработку эталона, распознавание пигментного новообразования, измеряет параметры новообразования, вычисляет вероятность малигнизации. 
Таблица 1. Клинические параметры пигментных новообразований кожи (могут быть измерены автоматически)

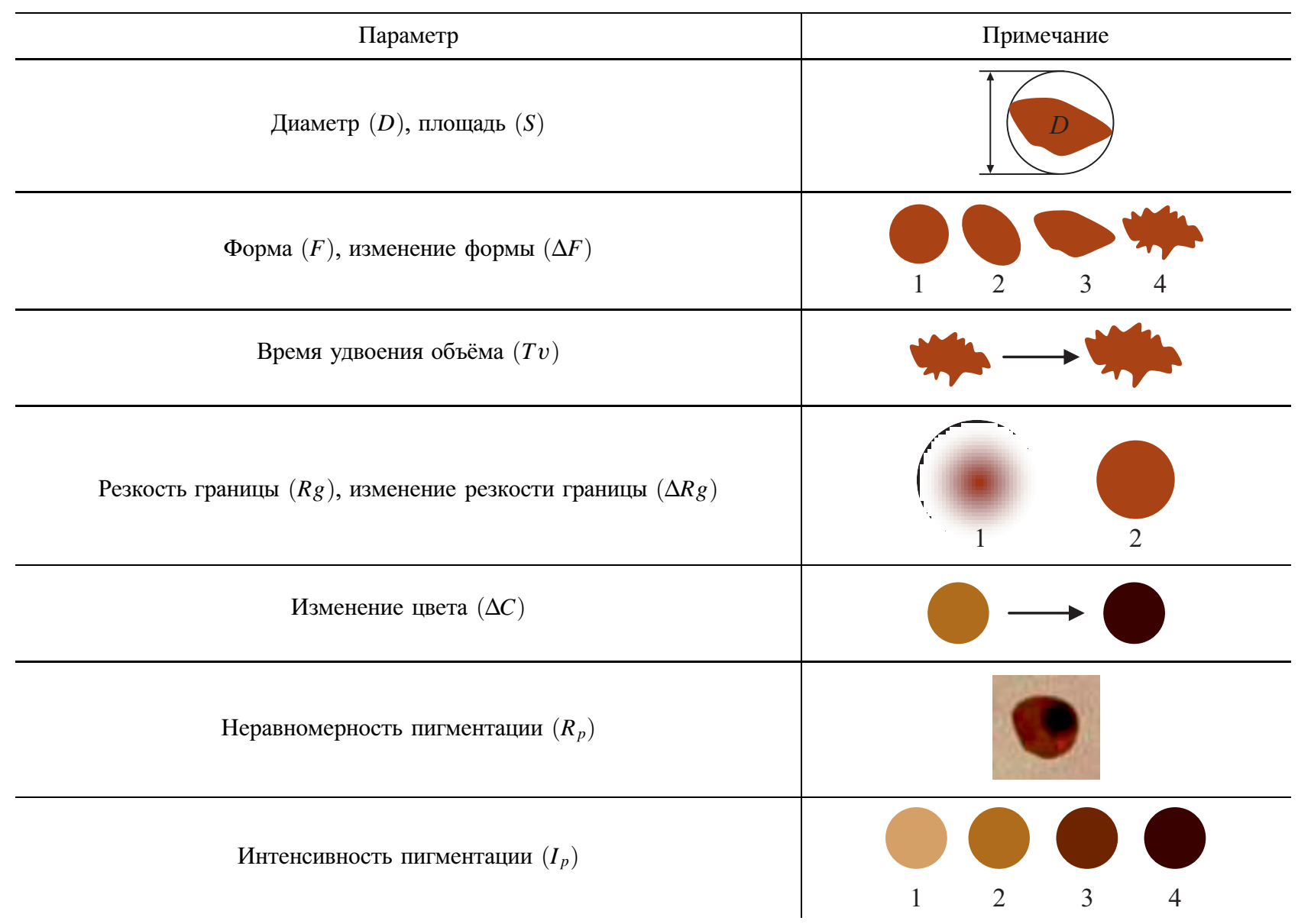

\section{2. Автоматизированная система скрининга пигментных новообразований кожи}

Методы и средства, позволяющие проводить скрининг пигментных новообразований кожи, помимо достаточной диагностической эффективности должны иметь достаточную скорость обследования, которую можно определить как площадь поверхности кожи, обследуемую в единицу времени. В этом аспекте среди существующих инструментальных средств наиболее эффективны для скрининга диагностические системы, позволяющие регистрировать цифровые изображения поверхности кожи целых анатомических областей с последующей цифровой обработкой изображений.

Автоматизированная система скрининга пигментных новообразований кожи, которую мы разработали [12,51], представляет собой сканер, который может регистрировать полноростовые изображения поверхности кожи, и анализирующее их специальное программное обеспечение (рис. 2). Специальное программное обеспечение устанавливают на персональный компьютер, к которому подключён сканер. Сканер имеет в своём составе цифровые фотокамеры, лампы-вспышки, систему контроля расстояния до пациента, систему управления. Матрицы цифровых фотокамер обеспечивают необходимое разрешение в предметной плоскости (не менее $6 \mathrm{pix} / \mathrm{mm}$ ). Предварительная юстировка обеспечивает соосность оптических осей объективов, а также их фокусировку в предметной плоскости. Лампы-вспышки позволяют создать стабильные условия освещения, необходимые для цветовых измерений. Система контроля расстояния до пациента помогает совместить пациента и предметную плоскость сканера.

Специальное программное обеспечение предназначено для скрининга и имеет следующие основные функции:

- регистрация изображений, управление сканером;

- картирование поверхности тела с автоматическим обнаружением новых, подозрительных и изменившихся невусов посредством измерения размеров, формы и цвета;

- режим живого просмотра при регистрации;

- коррекция позы пациента при повторной регистрации по ранее зарегистрированным изображениям;

- сравнение зарегистрированных изображений „бок о бок".

Такое построение автоматизированной системы скрининга пигментных новообразований кожи позволяет также проводить сравнительные обследования пациента, 
Таблица 2. Взаимосвязь комплекса клинических и измеряемых параметров

\begin{tabular}{|c|c|c|c|}
\hline \multirow[t]{2}{*}{ Параметр } & \multirow[t]{2}{*}{ Взаимосвязь } & \multicolumn{2}{|c|}{ Измеряемый параметр } \\
\hline & & Обозначение & Название \\
\hline$D$ & $D=L$ & $L$ & $\begin{array}{c}\text { Максимальное } \\
\text { расстояние между } \\
\text { точками контура }\end{array}$ \\
\hline$S$ & $S=S_{\text {н }}$ & $S_{\text {H }}$ & $\begin{array}{c}\text { Площадь за текущее } \\
\text { обследование }\end{array}$ \\
\hline \multirow[t]{2}{*}{$\overline{T_{v}}$} & \multirow{2}{*}{$T_{S}=\frac{T}{\frac{S_{\mathrm{H}}}{S_{\mathrm{c}}}-1}$} & $S_{\mathrm{c}}$ & $\begin{array}{c}\text { Площадь за предыдущее } \\
\text { обследование }\end{array}$ \\
\hline & & $T$ & $\begin{array}{c}\text { Период времени } \\
\text { между обследованиями }\end{array}$ \\
\hline \multirow[t]{3}{*}{$F$} & \multirow{3}{*}{$\begin{array}{ll}1, & E<P_{E}, \delta S_{E}<P_{\delta S_{E}}, \delta L<P_{\delta L} \\
2, & E \geq P_{E}, \delta S_{E}<P_{\delta S_{E}}, \delta L<P_{\delta L} \\
3, & \delta S_{E} \geq P_{\delta S_{E}}, \delta L<P_{\delta L} \\
4, & \delta L \geq P_{\delta L}\end{array}$} & $E$ & $\begin{array}{c}\text { Отношение максимального } \\
\text { расстояния к максимальному } \\
\text { поперечному расстоянию } \\
\text { между точками контура }\end{array}$ \\
\hline & & $\delta S_{E}$ & $\begin{array}{c}\text { Относительное отклонение } \\
\text { площади от площади } \\
\text { эллипса равных размеров }\end{array}$ \\
\hline & & $\delta L$ & $\begin{array}{c}\text { Относительное отклонение } \\
\text { длины контура } \\
\text { от длины сглаженного контура }\end{array}$ \\
\hline$\Delta F$ & $\begin{aligned} \Delta F= \begin{cases}1, & F=F_{c} \\
2, & F \neq F_{c}\end{cases} \\
F_{c}-\text { форма за предыдущее обследование }\end{aligned}$ & - & - \\
\hline$R_{g}$ & $R_{g}= \begin{cases}1, & \text { Grad } \geq P_{\mathrm{Grad}} \\
2, & \text { Grad }<P_{\mathrm{Grad}}\end{cases}$ & Grad & $\begin{array}{c}\text { Нормированный } \\
\text { максимальный } \\
\text { пограничный градиент } \\
\text { интенсивности } \\
\end{array}$ \\
\hline$\Delta R_{g}$ & $\begin{aligned} \Delta R_{g}=\left\{\begin{array}{l}1, \\
R_{g}=R_{g_{c}} \\
2, R_{g} \neq R_{g_{c}}\end{array}\right. \\
R_{g_{c}} \text { - резкость границы за предыдущее обследование }\end{aligned}$ & - & - \\
\hline$I_{p}$ & $I_{p}=\left\{\begin{array}{l}1: K<P_{1 K} ; 2: P_{1 K} \leq K<P_{2 K} \\
3: P_{2 K} \leq K<P_{3 K} ; \quad 4: K \geq P_{3 K}\end{array}\right.$ & $K$ & Контраст \\
\hline$R_{p}$ & $\begin{array}{c}S I=100 \% \frac{1}{3}\left(\frac{s r_{\mathrm{H}}}{r_{\mathrm{H}}}+\frac{s g_{\mathrm{H}}}{g_{\mathrm{H}}}+\frac{s b_{\mathrm{H}}}{b_{\mathrm{H}}}\right) \\
R_{p}= \begin{cases}1, & s I<P_{s I} \\
2, & s I \geq P_{s I}\end{cases} \end{array}$ & $s r_{\mathrm{H}}, s g_{\mathrm{H}}, s b_{\mathrm{H}}$ & $\begin{array}{l}\text { СКО координат цветности } \\
\text { за текущее обследование }\end{array}$ \\
\hline \multirow[t]{2}{*}{$\Delta C$} & $\delta C=100 \% \frac{1}{3}\left(\frac{\left|r_{\mathrm{H}}-r_{\mathrm{c}}\right|}{r_{r}}++\frac{\left|g_{\mathrm{H}}-g_{\mathrm{c}}\right|}{g_{c}}+\frac{\left|b_{\mathrm{H}}-b_{\mathrm{c}}\right|}{b_{c}}\right)$ & $r_{\mathrm{H}}, g_{\mathrm{H}}, b_{\mathrm{H}}$ & \multirow[t]{2}{*}{$\begin{array}{c}\text { Средние значения } \\
\text { координат цветности } \\
\text { за текущее и предыдущее } \\
\text { обследования }\end{array}$} \\
\hline & $\Delta C= \begin{cases}1, & \delta C<P_{\delta C} \\
2, & \delta C \geq P_{\delta C}\end{cases}$ & $r_{c}, g_{c}, b_{c}$ & \\
\hline
\end{tabular}



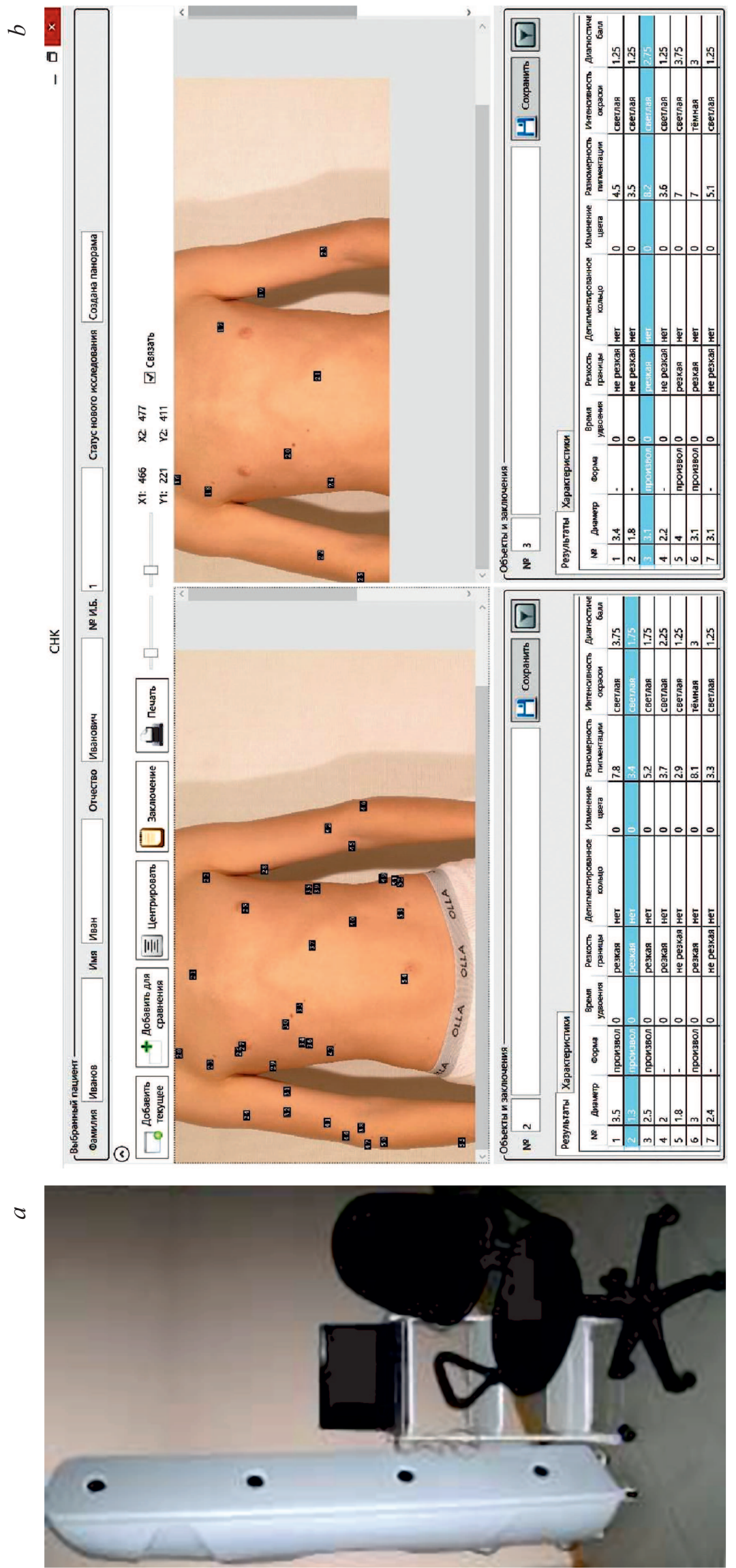

Рис. 2. Автоматизированная система скрининга пигментных новообразований кожи: $a-$ сканер и рабочее место, $b-$ специальное программное обеспечение. 
устанавливающие появление новых новообразований кожи и количественно оценивающие изменения имеющихся новообразований кожи.

\section{3. Автоматизированная термографическая система дополнительного обследования пигментных новообразований кожи}

Современный уровень элементной базы позволяет поновому взглянуть на применение термографии в медицине. Со времени первых экспериментов по регистрации термографических изображений существенно повысились эксплуатационные характеристики оборудования: габариты, масса, пространственная разрешающая способность, чувствительность. В своих исследованиях мы использовали портативный тепловизор Thermal Expert TE-V1 с объективом $8.5 \mathrm{~mm}$, который возможно подключать как к мобильным устройствам с операционной системой Android (смартфон, планшет), так и к персональному компьютеру. Характеристики тепловизора позволили регистрировать термографические изображения пигментных новообразований кожи (рис. 3). При этом диагностическим критерием является температурный контраст зоны пигментного новообразования и окружающих тканей.

\section{3. Обработка клинических изображений пигментных новообразований кожи}

Обработку клинических изображений пигментных новообразований кожи выполняли с применением единого подхода. После регистрации изображений программное обеспечение каждой системы выполняет оценку параметров качества изображений: яркость, контрастность, тоновая насыщенность и резкость [52,53]. Если качество изображения приемлемо, то алгоритм переходит к следующему этапу обработки. В случае мобильной системы выполняется нахождение элементов эталона и его параметров - значений цветов $R G B$ и масштаба изображения. По результатам цветокалибровки по эталону вычисляется вектор цветовой ошибки и по нему устраняется ошибка для каждого пикселя (вычитание). Геометрические отклонения, обусловленные неперпендикулярностью оптической оси камеры мобильного устройства к предметной плоскости, составляют $\pm 15 \%$. Эти искажения, вносимые неперпендикулярностью, легко устраняются с помощью аффинных преобразований [52,53]. В автоматизированной системе скрининга этот этап заменяется предварительной юстировкой. Затем происходит обнаружение границ новообразований. Ключевыми элементами сегментации являются метод наращивания областей и уточнение найденных границ морфологическими методами [52,53]. После устранения артефактов выполняется измерение размеров, формы и цвета каждого обнаруженного новообразования (табл. 2).
Таблица 3. Допустимые погрешности измерения параметров размеров, формы и цвета

\begin{tabular}{c|c|c}
\hline Измеряемый параметр & \multicolumn{2}{|c}{ Допустимая погрешность измерения } \\
\hline Обозначение & Формула & Значение \\
\hline$L$ & $\Delta_{L}=\frac{L \Delta T_{S}}{2 T_{S}} \sqrt{\frac{\left|T^{2}-T_{S}^{2}\right|}{T^{2}+T_{S}^{2}}}$ & $0.45 \mathrm{~mm}$ \\
$S_{\mathrm{H}}$ & $\Delta_{S}=\frac{S_{t}-S_{t-T}}{T} \sqrt{\frac{\Delta_{T_{S}}^{2}\left(S_{t}-S\right)}{S_{t-T}^{2}+S_{t}^{2}}}$ & $2.9 \mathrm{~mm}^{2}$ \\
$S_{c}$ & $\Delta_{T}$ & \\
$T$ & $\Delta_{E}=\frac{\Delta_{L}}{L} \cdot \sqrt{2}$ & $0.5 \mathrm{day}$ \\
$E$ & $\Delta_{\delta S}=\frac{\Delta T_{S}}{T} \delta S^{\mid} \sqrt{\left|\delta S^{2}-1\right|}$ & 0.117 \\
$\delta S_{E}$ & $\Delta_{\delta L}=\frac{\Delta_{L}}{L} \cdot \sqrt{2}$ & 0.30 \\
$\delta L$ & $\Delta_{G}=\frac{\Delta_{L}}{\Delta X^{2}}$ & $7.5 \%$ \\
Grad & & $10 \%$ \\
$K$ & Результаты исследований & \\
$s r_{\mathrm{H}}, s g_{\mathrm{H}}, s b_{\mathrm{H}}$ & & \\
$r_{\mathrm{H}}, g_{\mathrm{H}}, b_{\mathrm{H}}$ & & \\
$r_{c}, g_{c}, b_{c}$ & &
\end{tabular}

\section{4. Погрешности морфометрии клинических изображений пигментных новообразований кожи}

Масштаб регистрируемых изображений случайным образом искажается при взаимном позиционировании устройства регистрации изображений и объекта регистрации. При использовании мобильных устройств (смартфон, планшет) и эталона на этапе обработки изображений возможна коррекция вносимых искажений. В автоматизированной системе скрининга искажения минимизируют юстировкой.

В то же время есть дополнительные факторы, влияющие на процесс регистрации: динамика вертикальной позы пациента и дыхание пациента, позиционирование мобильного устройства. Эти факторы случайным образом искажают масштаб регистрируемых изображений и приводят к погрешностям измерения линейных размеров и площади. В теории фотограмметрии [54] проработан математический аппарат оценки масштаба изображения в зависимости от взаимного положения в пространстве регистрирующего устройства и регистрируемого объекта. С помощью этого математического аппарата можно определить случайные погрешности измерения, связанные с изменениями масштаба изображения.

Искажения масштаба регистрируемых изображений, обусловленные изменением угла положения регистрируемого объекта в пространстве относительно регистрирующего устройства $(\theta)$, описываются соотношениями

$$
\begin{aligned}
F\left(k_{\theta}, c_{\theta}\right)= & k_{\theta}^{2} \max _{\varphi}\left[\left(k_{\theta} \cos (\varphi)\right.\right. \\
& \left.\left.+c_{\theta} \sin (\varphi)\right)^{2}+\sin (\varphi)^{2}\right]^{-\frac{1}{2}}-1,
\end{aligned}
$$



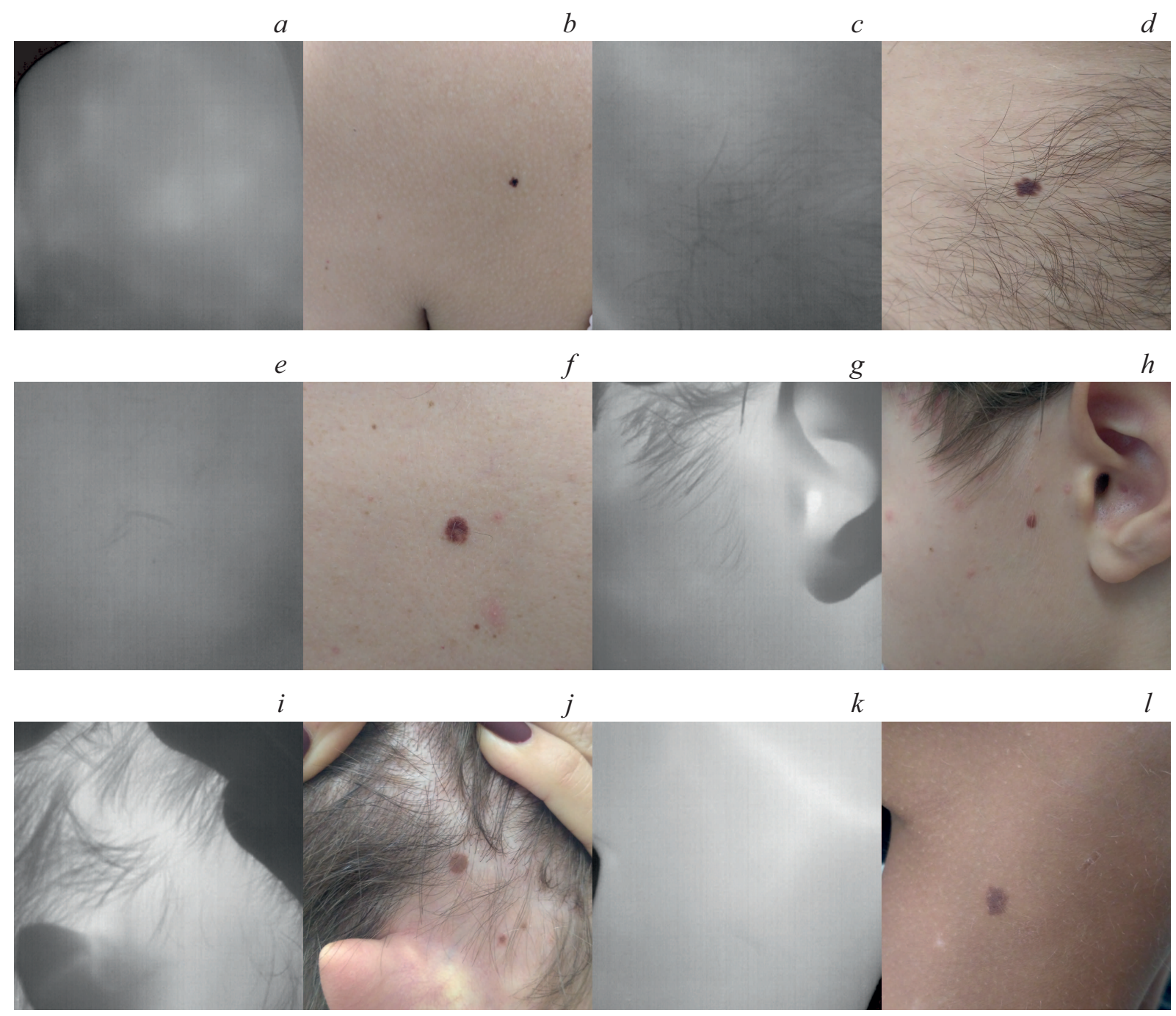

Рис. 3. Примеры термографических и соответствующих им клинических изображений пигментных новообразований кожи, температурный контраст отсутствует: $a, c, e, g, i, k-$ термографические изображения; $b, d, f, h, j, l-$ соответствующие им клинические изображения.

где $k_{\theta}=\cos (\theta)-\frac{x}{f} \sin (\theta), c_{\theta}=\frac{y}{f} \sin (\theta), x$ и $y-$ координаты точки в кадре, $f$ - фокусное расстояние объектива.

В работе [12] мы оценивали искажения масштаба и связанные с этим погрешности измерения. Суммарная погрешность измерения линейных размеров не превышает $2.8 \%$, суммарная погрешность измерения площади не превышает 4.0\%. При этом допускается позиционирование пациента в предметной плоскости в некоторых пределах (для автоматизированной системы скрининга - до $77 \mathrm{~mm}$ ). Требуемая точность для угла места и азимутального угла ориентации регистрирующего устройства составляет $9.2^{\circ}$. Указанные требования достаточно легко соблюсти при юстировке или при калибровке по эталону: результаты измерения параметров размеров и формы останутся в пределах допустимых погрешностей.

\section{5. Вычислительная диагностика пигментных новообразований кожи}

В дерматологической практике при диагностике пигментных новообразований кожи обычно используют алгоритм $A B C D$ [55-57] (есть варианты для визуального и дерматоскопического обследования) или другие похожие алгоритмы. Каждому визуальному признаку врач назначает балл в соответствии с алгоритмом. Оценивают: $A-$ асимметрию, $B$ - изрезанность границы, $C-$ изменение цвета, $D$ - изменение диаметра. Получившаяся сумма баллов сравнивается с пороговыми значениями. Итогом является диагноз: невус, атипичный невус, меланома.

Существует программное обеспечение, автоматизирующее подсчёт суммарного балла или же анализирующее дерматоскопические изображения и дающее врачу второе мнение. 
Мы придерживаемся подхода автоматизации диагностического процесса и в отличие от большинства аналогов автоматизируем как оценку клинических параметров, так и итоговую оценку пигментных новообразований. Вся получаемая диагностическая информация доступна врачу. Итоговая оценка пигментных новообразований выполняется методами вычислительной диагностики, а именно посредством машинного обучения. Была создана верифицированная база клинических изображений пигментных новообразований кожи: 360 изображений высокого разрешения (для мобильной системы скрининга), 680 изображений низкого разрешения (не менее $6 \mathrm{pix} / \mathrm{mm}$, для автоматизированной системы скрининга). Верификация выполнялась путём гистологического исследования пигментных новообразований кожи, удаляемых по медицинским показаниям.

Далее для каждого изображения определяли комплекс клинических параметров и исследовали законы распределения полученных данных в целом и для классов новообразований в частности: пигментный невус, атипичный невус (с лентигинозной меланоцитарной дисплазией), меланома. Законы распределения не соответствовали нормальному распределению, поэтому использовали Бокс-Кокс преобразование [58]. Это позволило использовать метод главных компонент для оптимизации пространства признаков. Сложность конфигурации разделяющих классы поверхностей обусловило использование метода $k$-ближайших соседей $(k-\mathrm{NN})$. При обучении классификатора выполнялась оптимизация по числу соседей и варианту модификации (объединённая и попарная модификации), а также по разбиению верифицированных данных на обучающую и контрольную выборки.

Автоматизированная термографическая система использовалась для проверки уровня метаболизма в области новообразований кожи, классифицированных как атипичный невус или меланома. Так, в случае меланомы в качестве критерия используют повышение температуры опухоли по сравнению с окружающими тканями на 0.5 и более градусов. При этом для меланом характерно повышение температуры на $0.7-1.4^{\circ} \mathrm{C}$ (и более), для доброкачественных новообразований температура обычно ниже на $1.5^{\circ} \mathrm{C}$ или незначительно выше: до $0.5^{\circ} \mathrm{C}[59-61]$.

\section{6. Апробация}

Апробация методики обследования с применением экспериментального образца мобильной системы скрининга пигментных новообразований кожи в условиях клиники представлена в нашей работе [50]. Для исследования были отобраны три тестовые группы гистологически верифицированных изображений пигментных новообразований кожи с подтверждённым диагнозом: группа с изображениями доброкачественных новообразований (165 изображений), группа риска (185 изображений атипичных невусов) и злокачественные новообразования (10 изображений). Диагностическая чувствительность метода составила 97\%, специфичность - 95\%.

Апробация методики обследования с применением экспериментального образца автоматизированной системы скрининга пигментных новообразований кожи представлена в наших работах $[51,62]$. Было обследовано 570 пациентов. Из них у 161 пациента были удалены пигментные новообразования кожи, преимущественно с клиническим диагнозом меланома или диспластический невус (с подозрением на тяжёлую степень дисплазии). По результатам гистологической верификации чувствительность методики составила 97\%, специфичность $92 \%$.

\section{7. Обсуждение}

Современной тенденцией в ранней диагностике меланомы является обследование пигментных новообразований кожи малого диаметра, что может способствовать вторичной профилактике меланомы и дальнейшему снижению показателей смертности от неё [63-66]. Вместе с тем известно, что чувствительность и специфичность клинической диагностики меланомы варьирует в зависимости от размеров пигментного новообразования: ниже при диаметрах до $5 \mathrm{~mm}$ и выше при диаметpax свыше $5 \mathrm{~mm}$, что установлено гистологическими методами [67]. В то же время по различным причинам объективного и субъективного характера могут возникать ложноотрицательные и ложноположительные ошибки при проведении гистологических исследований пигментных новообразований, достигающие $85.7 \%$ при диаметре менее $6 \mathrm{~mm}$ [63]. Проблему ранней диагностики меланомы малого диаметра возможно решать с помощью различных средств инструментальной диагностики. Однако большинство разработанных методов и средств требуют специальных знаний и высокой квалификации диагноста (дерматоскопия, магнитно-резонансная микроскопия, конфокальная лазерная микроскопия, оптическая когерентная томография, ультразвуковое сканирование кожи, флуоресцентная диагностика ит.д.). Существуют перспективные методы, которые в ходе пилотных исследований демонстрируют обнадёживающие результаты (конфокальная лазерная микроскопия, оптическая когерентная томография, спектроскопия и визуализация в терагерцовом диапазоне). Конфокальная лазерная микроскопия, оптическая когерентная томография могут стать неинвазивными аналогами гистологического исследования в случае диагностики новообразований малой (до $2 \mathrm{~mm}$ ) толщины, если будет накоплена соответствующая экспериментальная база. Спектроскопия и визуализация в терагерцовом диапазоне могут послужить основной для перспективных инструментальных средств дифференциальной диагностики пигментных новообразований кожи, так как в настоящее время установлены особенности оптических терагерцовых характеристик различных 
классов новообразований [39]. Появление суперразрешающих систем визуализации в терагерцовом диапазоне на основе спектроскопического контрастирования вполне способно создать тренд в развитии диагностики опухолей малого диаметра, о чём свидетельствуют современные исследования $[68,69]$. Возможно, имеющиеся ограничения разрешающей способности такого оборудования, способного визуализировать структуры размерами в доли миллиметра, будут компенсированы именно высокой чувствительностью к спектральным различиям биологических тканей в норме и при патологии. Также предстоит разрабатывать такую конструкцию оборудования, которая бы позволяла удобно и быстро обследовать новообразования in vivo.

Проблема ранней диагностики меланомы, в том числе малого диаметра, может решаться также путём динамического наблюдения пигментных новообразований кожи. Меланома кожи по крайней мере в 70\% случаев имеет фазу горизонтального роста (поверхностно распространяющаяся меланома) [47], что может быть зафиксировано как визуально (в том числе самим пациентом), так и с использованием инструментальных средств. При этом наиболее очевидными и легко фиксируемыми являются изменения таких клинических параметров, как размеры и площадь новообразования, а также параметров цвета. Именно поэтому онкологи рекомендуют пациентам группы риска регулярно (не реже раза в год) проходить обследование кожных покровов, позволяющее выявить новые и изменяющиеся новообразования. Использование предлагаемого нами подхода увеличивает эффективность таких обследований за счёт устранения субъективных ошибок и количественной оценки клинических параметров новообразований кожи, что способствует выявлению меланом на ранней стадии в горизонтальной фазе роста.

\section{Заключение}

Предложен и апробирован комплексный подход к ранней диагностике меланомы кожи. Подход состоит в поэтапном использовании нескольких методов обследования пигментных новообразований кожи: самостоятельный скрининг с помощью мобильного устройства (смартфон, планшет) со специальным программным обеспечением, автоматизированный скрининг методом картирования, дополнительное термографическое обследование.

Методы объединяет как общий объект исследования, так и принципы построения и реализации изображающих систем и специального программного обеспечения. Диагностическая задача решается как задача измерения клинических параметров новообразований кожи с последующей классификацией полученных данных. Ключевым аспектом является метрологическое обеспечение измерений. Установлено, что погрешности измерений выбранными методами не превышают допустимые погрешности при соблюдении условий проведения изме- рений. Для классификации используется метод $k$-ближайших соседей, обеспечивающий построение сложных поверхностей разделения классов. Результаты каждого диагностического канала могут быть учтены при постановке диагноза, что повышает эффективность диагностического процесса. Апробация показала высокие чувствительность и специфичность предложенного подхода, превышающие $90 \%$.

\section{Финансирование работы}

Разработка и апробация экспериментального образца мобильной системы скрининга пигментных новообразований кожи поддержаны Российским фондом фундаментальных исследований (РФФИ), проект № 18-38-00958. Разработка метрологического обеспечения и исследования по термографии пигментных новообразований кожи поддержаны РФФИ, проект № 18-29-02099.

Работа также была поддержана проектом повышения конкурентоспособности ведущих российских университетов ,5-100“ Первого Московского государственного медицинского университета им. И.М. Сеченова.

\section{Соблюдение этических стандартов}

Все исследования и процедуры с участием людей соответствуют этическим стандартам институционального и/или национального комитета по исследовательской этике и Хельсинкской декларации 1964 г. и ее последующим изменениям или сопоставимым нормам этики. От каждого из включенных в исследование участников было получено информированное добровольное согласие.

\section{Конфликт интересов}

Авторы заявляют, что у них нет конфликта интересов.

\section{Список литературы}

[1] Eisemann N., Waldmann A., Geller A.C., Weinstock M.A., Volkmer B., Greinert R., Breitbart E.W., Katalinic A. // J. Invest. Dermatol. 2014. V. 134. N 1. P. 43. doi 10.1038/jid.2013.304.

[2] LeBoit P.E., Burg G., Weedon D., Sarasain A. World Health Organization Classification of Tumours. Pathology and Genetics of Skin Tumours. IARC Press: Lyon, 2006. 355 p.

[3] Shaikh WR, Xiong M. Weinstock M.A. // Arch. Dermatol. 2012. V. 148. P. 30. doi 10.1001/archdermatol.2011.264

[4] Chen S.T., Geller A.C., Tsao H. // Curr. Dermatol. Rep. 2013. V. 2. N 1. P. 24. doi 10.1007/s13671-012-0035-5

[5] Nikolaou V., Stratigos A.J. // Br. J. Dermatol. 2014. V. 170. N 1. P. 11. doi $10.1111 / \mathrm{bjd} .12492$

[6] Tsao H., Olazagasti J.M., Cordoro K.M., Brewer J.D., Taylor S.C., Bordeaux J.S., Chren M.M., Sober A.J., Tegeler C., Bhushan R., Begolka W.S. // J. Amer. Acad. Dermatol. 2015. V. 72. N 4. P. 717. doi 10.1016/j.jaad.2015.01.025

[7] Siegel R.L., Miller K.D., Jemal A. // CA Cancer J. Clinicians. 2016. V. 66. N 1. P. 7. doi 10.3322/caac. 21332 
[8] Siegel R.L., Miller K.D., Jemal A. // CA Cancer J. Clinicians. 2017. V. 67. N 1. P. 7. doi 10.3322/caac.21387

[9] Rahib L., Smith B.D., Aizenberg R., Rosenzweig A.B, Fleshman J.M., Matrisian L.M. // Cancer Research. 2014. V. 74. N 11. P. 2913. doi 10.1158/0008-5472.CAN-14-0155

[10] Конопацкова О.М., Жандарова Л.Ф. // Тез. Всесоюз. симпоз. 1990. С. 33.

[11] Mayer J.E., Swetter S.M., Fu T., Geller A.C. // J. Amer. Acad. Dermatol. 2014. V. 71. N 4. P. 599.e1. doi 10.1016/j.jaad.2014.05.046

[12] Kudrin K.G., Matorin O.V., Reshetov I.V. // J. Phys.: Conf. Ser. 2015. V. 584. P. 012001. doi 10.1088/17426596/584/1/012001

[13] Eggermont A., Spatz A., Robert C. // The Lancet. 2014. V. 1. N 383(9919). P. 816. doi 10.1016/S0140-6736(13)60802-8

[14] Rubegni P., Burroni M., Perotti R., Fimiani M., Andreassi L., Cevenini G., Barbini P. // J. Investigative Dermatology. 2002. V. 119. P. 471.

[15] Bafounta M.L., Beauchet A., Aegerter P., Saiag P. // Arch. Dermatol. 2001. V. 137. P. 1343.

[16] Cohen D.E., Sangueza O.P., Pass E., Stiller M.J. // Int. J. Dermatol. 1993. V. 32. P. 257. doi 10.1111/j.13654362.1993.tb04263.x

[17] Bleve M., Capra P., Pavanetto F., Perugini P. // Dermatology Research and Practice. 2012. P. 673706. doi 10.1155/2012/673706

[18] Demidov L.V., Sokolov D.V, Bulycheva I.V. // J. N.N. Blokhin Russian Cancer Research Center RAMS. 2007. V. 18. N 1. P. 36.

[19] Kittler H., Marghoob A.A., Argenziano G. // J. Amer. Acad. Dermatol. 2016. V. 74. N 6. P. 1093. doi 10.1016/j.jaad.2015.12.038

[20] Rosendahl C., Hishon M., Cameron A., Barksdale S., Weedon D., Kittler H. // Dermatology Practical and Conceptual. 2014. V. 4. N 2. P. 69. doi 10.5826/dpc.0402a15

[21] Deinlein T., Richtig G., Schwab C., Scarfi F., Arzberger E., Wolf I., Hofmann-Wellenhof R., Zalaudek I. // J. German Soc. Dermatol. 2016. V. 14. N 2. P. 144. doi 10.1111/ddg.12903

[22] Bleve M., Capra P.F., Perugini P. // Magnetic Resonance in Medicine. 1997. V. 37. N 2. P. 185.

[23] Rajadhyaksha M., González S., Zavislan J.M., Anderson R.R., Webb R.H. // J. Investigative Dermatology. 1999. V. 113. P. 293. doi 10.1046/j.1523-1747.1999.00690.x

[24] Gonzalez S. // Actas Dermosifiliograficas. 2009. V. 100. N 2. P. 59.

[25] Longo C., Ragazzi M., Ragazzi M., Nehal K., Bennassar A., Pellacani G., Guilera J.M. // Dermatologic Clinics. 2016. V. 34. N 4. P. 497. doi 10.1016/j.det.2016.05.012

[26] Pellacani G., De Pace B., Reggiani C., Cesinaro A.M., Argenziano G., Zalaudek I., Soyer H.P., Longo C. // Experimental Dermatology. 2014. V. 23. N 6. P. 414. doi 10.1111/exd.12417

[27] Rollins A.M., Kulkarni M.D., Yazdanfar S., Ung-arunyawee R., Izatt J.A. // Optics Express. 1998. V. 3. P. 219. doi 10.1364/OE.3.000219

[28] Saxer C.E., de Boer J.F., Park H.B., Zhao Y., Chen Z., Nelson J.S. // Opt. Lett. 2000. V. 25(18). P. 1355. doi 10.1364/OL.25.001355

[29] Olsen J., Themstrup L., Jemec G.B. // G. Ital. Dermatol. Venereol. 2015. V. 150. N 5. P. 603.

[30] MacKinnon N., Vasefi F., Booth N., Farkas D.L. // Proc. SPIE. 2016. V. 9711. N 1. P. 971111. doi 10.1117/12.2222415
[31] Zhao1 B., He Y. // Exp. Rev. Anticancer Therapy. 2010. V. 10. P. 1797. doi 10.1586/era.10.154

[32] Rohrbach D.J., Muffoletto D., Huihui J., Saager R. Keymel K., Paquette A., Morgan J., Zeitouni N., Sunar U. // Academic Radiology. 2014. V. 21. N 2. P. 263. doi 10.1016/j.acra.2013.11.013

[33] Neuschmelting V., Burton N.C., Lockau H., Urich A., Harmsen S., Ntziachristos V., Kirchera M.F. // Photoacoustics. 2016. V. 4. N 1. P. 1. doi 10.1016/j.pacs.2015.12.001

[34] Müller J., Hartmann J., Bert C. // Phys. Med. Biol. 2016. V. 61. N 7. P. 2646. doi 10.1088/0031-9155/61/7/2646

[35] Petersen B., Philipsena P.A., Wulf H.C. // Photochem. Photobiol. Sci. 2014. V. 13. N 8. P. 1123. doi 10.1039/C4PP00066H

[36] Faust O., Acharya U.R., Ng E.Y.K., Hong T.J., Yu W. // Infrared Phys. Technol. 2014. V. 66. P. 160. doi 10.1016/j.infrared.2014.06.001

[37] North J.P., Garrido M.C., Kolaitis N.A., LeBoit P.E. // Amer. J. Surg. Pathol. 2014. V. 38. N 6. P. 824. doi 10.1097/PAS.0000000000000189

[38] Borisova E.G., Angelova L.P., Pavlova E.P. // IEEE J. Selected Topics in Quant. Electron. 2014. V. 20. N 2. P. 211. doi 10.1109/JSTQE.2013.2280503

[39] Zaytsev K.I., Kudrin K.G., Karasik V.E., Reshetov I.V., Yurchenko S.O. // Appl. Phys. Lett. 2015. V. 106. N 5. P. 053702. doi 10.1063/1.4907350

[40] Зайцев К.И., Черномырдин Н.В., Кудрин К.Г., Решетов И.В., Юрченко С.О. // Опт. и спектр. 2015. Т. 119. № 3. C. 404-410. doi 10.7868/S0030403415090305

[41] Ganster H., Pinz P., Rohrer R., Wildling E., Binder M., Kittler H. // IEEE Transactions on Medical Imaging. 2001. V. 20. N 3. P. 233. doi $10.1109 / 42.918473$

[42] Masood A., Al-Jumaily A.A. // Int. J. Biomed. Imaging. 2013. V. 2013. P. 1. doi $10.1155 / 2013 / 323268$

[43] Isasi A. Gola, Zapirain García B., Méndez Zorrilla A. // Computers in Biology and Medicine. 2011. V. 41. N 9. P. 742. doi 10.1016/j.compbiomed.2011.06.010

[44] Ахмедов Б.П., Хасанова 3.М. // Современная онкология. 2002. T. 4. № 4. C. 50.

[45] Patel S., Eluri M., Boyers L.N., Karimkhani C., Dellavalle R. // Dermatol Online J. 2015. V. 21. N 2.

[46] Кудрин К.Г. // Онкохирургия. 2014. .1. Т. 6. С. 71.

[47] Wolff K., Goldsmith L., Katz S., Gilchrest B., Paller A.S., Leffell D. Fitzpatrick's Dermatology in General Medicine. 7th Edition. N.Y:: McGraw-Hill, 2008.

[48] Чиссов В.И., Старинский В.В., Александрова Л.М. и др. Раннее выявление и профилактика меланомы кожи. М.: ФГБУ „МНИОИ им. П.А. Герцена“ Минздрава России. 2013. 22 c.

[49] Deshin I., Rimskaya E., Apollonova I., Kudrin K., Nursultanova Z., Nikolaev A. // AIP Conf. Proc. 2019. V. 2140. P. 020017. doi 10.1063/1.5121942

[50] Римская Е.Н., Щадько А.О., Аполлонова И.А., Николаев А.П., Брико А.Н., Дешин И.А., Бережсной П.Ю., Кудрин К.Г., Зайцев К.И., Тучин В.В., Решетов И.В. // Опт. спектр. 2019. Т. 126. В. 5. С. 584.

doi 10.21883/OS.2019.05.47657.6-19; Rimskaya E.N., Schadko A.O., Apollonova I.A., Nikolaev A.P., Briko A.N., Deshin I.A., Bereshnoy P.U., Kudrin K.G., Zaytsev K.I., Tuchin V.V., Reshetov I.V. // Opt. Spectrosc. 2019. V. 126. P. 503. doi 10.1134/S0030400X19050230

[51] Кудрин К.Г., Маторин О.В., Решетов И.В. // Голова и шея. 2014. № 1. С. 9. 
[52] Gonzalez R.C., Woods R.E. Digital Image Processing Using MATLAB. Pearson Prentice Hall, 2003. 609 p.

[53] Gonzalez R.C., Woods R.E. Digital Image Processing. Pearson Prentice Hall, 2004. 344 p.

[54] Агапов С.В. Фотограмметрия сканерных снимков. М.: Картгеоцентр-Геодезиздат, 1996. 76 с.

[55] Bazeille S., Quidu I., Jaulin L., Malkasse J.-P. // CMM. France. 2006. P. 16.

[56] Rigel D.S., Russak J., Friedman R. // CA: A Cancer J. Clinicians. 2010. V. 60. N 5. P. 301. doi 10.3322/caac.20074

[57] Glazer A.M., Farberg A.S., Winkelmann R.R., Tucker N., Rigel D.S. // Dermatology Online J. 2017. V. 23. N 8. P. 13030/qt9xx2907s.

[58] Kutner M., Nachtsheim C., Neter J., Li W. Appl. Linear Statistical Models. Homewood, IL: McGraw-Hill/Irwin, 2004.

[59] Cetingul M.P., Herman C. // Phys. Med. Biol. 2010. 7;55(19):5933-51. doi 10.1088/0031-9155/55/19/020

[60] Arumi-Uria M., McNutt N.S., Finnerty B. // Mod. Pathology. 2003. V. 16. P. 764.

[61] Barnhill R.L., Fine J.A., Roush G.C., Berwick M. // Cancer. 1996. V. 78. P. 427.

[62] Кудрин К.Г., Маторин О.В., Решетов И.В. // Онкохирургия. 2013. № 1. Т. 5. С. 8.

[63] Сергеев Ю.Ю., Мордовцева В.В. // Кремлевская медицина. Клинический вестник. 2018. № 1. С. 84.

[64] Moreno-Ramirez D., Ojeda-Vila T., Rios-Martin J.J., NietoGarcia A., Ferrandiz L. // Melanoma Res. 2015. V. 25. P. 450. doi 10.1097/CMR.0000000000000184

[65] Akay B.N., Okcu Heper A., Clark S., Erdem C., Rosendahl C.O., Kittler H. // Int. J. Dermatol. 2017. N 56. P. 1498. doi 10.1111/ijd.13728

[66] Seidenari S., Ferrari C., Borsari S., Fabiano A., Bassoli S., Giusti F., Ponti G., Magnoni C. // Br. J. Dermatol. 2014. V. 171. P. 1006. doi 10.1111/bjd.12542

[67] Black W.C., Hunt W.C. // Am. J. Surg. Pathol. 1990. N 14. P. 44-52.

[68] Chernomyrdin N.V., Kucheryavenko A.S., Kolontaeva G.S., Katyba G.M., Karalkin P.A., Parfenov V.A., Gryadunova A.A., Norkin N.E., Smolyanskaya O.A., Minin O.V., Minin I.V., Karasik V.E., Zaytsev K.I. // Proc. SPIE. 2018. 10677, Unconventional Optical Imaging, 106771Y (24 May). doi $10.1117 / 12.2306132$

[69] Nguyen Pham H.H., Hisatake S., Minin O.V., Nagatsuma T., Minin I.V. // APL Photonics. 2017. V. 2. N 5. P. 056106. doi $10.1063 / 1.4983114$ 